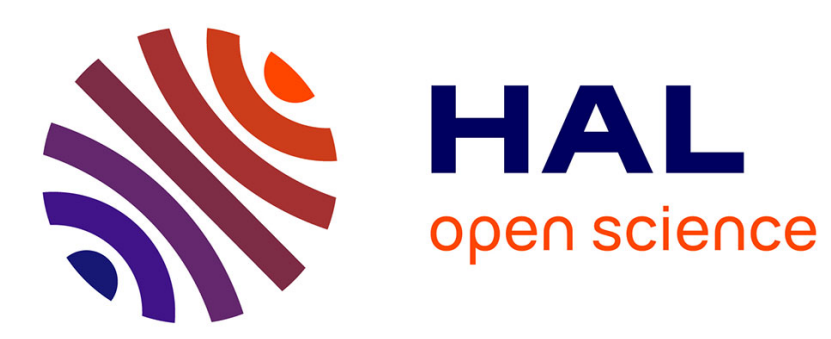

\title{
Modeling dependent competing failure processes based on stochastic hybrid systems
}

Mengfei Fan, Rui Kang, Zhiguo Zeng, Enrico Zio

\section{To cite this version:}

Mengfei Fan, Rui Kang, Zhiguo Zeng, Enrico Zio. Modeling dependent competing failure processes based on stochastic hybrid systems. 26th European Safety and Reliability Conference, ESREL 2016, Sep 2016, glasgow, United Kingdom. pp.410. hal-02447686

\section{HAL Id: hal-02447686 \\ https://hal.science/hal-02447686}

Submitted on 24 Feb 2020

HAL is a multi-disciplinary open access archive for the deposit and dissemination of scientific research documents, whether they are published or not. The documents may come from teaching and research institutions in France or abroad, or from public or private research centers.
L'archive ouverte pluridisciplinaire HAL, est destinée au dépôt et à la diffusion de documents scientifiques de niveau recherche, publiés ou non, émanant des établissements d'enseignement et de recherche français ou étrangers, des laboratoires publics ou privés. 


\title{
Modeling dependent competing failure processes based on stochastic hybrid systems
}

\author{
Mengfei Fan \& Rui Kang \\ Center for Resilience and Safety of Critical Infrastructures, Beihang University, Beijing, China
}

Zhiguo Zeng \& Enrico Zio

Chair System Science and the Energy Challenge, Fondation Electricite de France (EDF), CentraleSuplec, Universit Paris-Saclay, Grande Voie des Vignes, 92290 Chatenay-Malabry, France

Energy Department, Politecnico di Milano, Milano, Italy

\begin{abstract}
A novel approach is developed to calculate the reliability of dependent competing failure processes (DCFP) based on stochastic hybrid systems (SHS). The DCFP considers the dependency between a degradation process and a random shock process, where the arrival of a random shock will bring an abrupt change to the degradation process. A SHS model is developed to describe the evolution of system behaviors, where the degradation process is characterized by stochastic differential equations (SDEs). The SDEs are dependent on the transition of system modes, which represent the random shock process. The conditional moments of the degradation measure in each system mode is derived. Based on the conditional moments, the system reliability and its lower bound are estimated using First Order Second Moment (FOSM) method and Markov's inequality, respectively. Results show that the SHS-based method yields an accurate estimation of reliability with less computational costs compared to traditional Monte Carlo-based method.
\end{abstract}

\section{INTRODUCTION}

Industrial components, systems and products might fail due to multiple failure processes, i.e. wear, corrosion, fracture, fatigue and etc. (Jiang et al., 2015). In literature, failure processes are categorized into two types: degradation processes (or soft failures) and catastrophic failure processes (or hard failures) (Li and Pham, 2005). In general, soft failures are modeled by a continuous degradation process, e.g., diffusion process (Lemoine and Wenocur, 1985), continuous-time semi Markov's process (Lin et al., 2015), etc. Hard failures are modeled by a random shock process, e.g., a Poisson process (Lemoine and Wenocur, 1985, Lin et al., 2015, Klutke and Yang, 2002). Soft failures occur when the total degradation exceeds its threshold. Hard failures are caused by the random shock process in various shock patterns such as extreme shock, cumulative shocks, $\delta$-shocks, $m$-shocks, run shocks (Rafiee et al., 2014).

In practice, those processes are often dependent, making the reliability modeling more complex and therefore referred to as multiple Dependent Competing Failure Processes (DCFP). As a result of the dependence, the arrival shocks may change some attributes of the degradation process (Rafiee et al., 2014) or bring additional damages to the degradation process (Peng et al., 2010, Jiang et al., 2012). The hard failure process may be effected by the degradation process in terms of shock intensity (Caballe et al., 2015, Huynh et al., 2012, Huynh et al., 2011, Bagdonavicius et al., 2004), shock loads (Fan et al.,
2000), hard failure threshold (Jiang et al., 2012) and etc. Various models are developed to calculate the reliability of the DCFPs. For example, Peng et al. (2010) assume that the arrival of shocks would bring an abrupt increase to the normal degradation process and develop a model to calculate the reliability. Jiang et al. (2012) develop a reliability model to consider the dependency between degradation and shocks where the failure threshold is shifted by random shocks. Rafiee et al. (2014) consider the circumstance where the degradation rate is shifted by different shock patterns. Jiang et al. (2015) categorize shocks into different shock zones based on their magnitudes, and consider dependence where shocks in different zones have different effects on the degradation process.

In the existing reliability models concerning DCFP, systems fail when either a soft failure or a hard failure happens. Hence, in those models, system's reliability is regarded as the probability that neither soft failure nor hard failure occurs in a period of time, which is calculated by the product of the conditional probability of no soft failure and that of no hard failure conditioned on parameters shared by the two probability functions. Due to the introduction of dependence, the developed reliability functions are usually too complicated to be evaluated analytically. Rather, the reliability has to be calculated by Monte Carlo method, which needs large amount of computation to ensure the accuracy of the results. 
Stochastic Hybrid System (SHS) models are capable of characterizing the system behaviors that involve both continuous and discrete stochastic processes (Hespanha, 2004). Using Dynkin's formula, the conditional moments of each system state of an SHS can be determined by solving a set of differential equations (Hespanha, 2004). In this paper, we try to exploit the SHS model to reduce the computational costs of calculating the reliability of DCFPs. As an initial attempt, we consider the system discussed by Peng et al. (2010) in this paper, where the degradations depend on random shocks, i.e., the arrival of a random shock would cause an abrupt increase on the cumulative degradation measures.

The reminder of this paper is organized as follows. Sect. 2 gives a brief introduction of stochastic hybrid systems and its solutions. Sect. 3 formulates the DCFP as an SHS and derives the conditional moments of the system states based on Dynkin's formula. The conditional moments are used in Sect. 4 to estimate the reliability and its lower bound for the DCFP. Sect. 5 presents a numerical example to demonstrate the proposed model and compares its solution with the results from Monte Carlo simulation. A brief conclusion of the this paper is made in Sect. 6.

\section{BACKGROUND KNOWLEDGE ON SHS}

Generally speaking, stochastic hybrid systems are suitable to characterize systems whose state evolution contains both continuous stochastic processes and discrete stochastic processes. A latest formally defined SHS model proposed by Hespanha (2004) is applied in this paper. This SHS model is a subset of a more general class of stochastic processes referred to as the piecewise-deterministic Markov's processes (Davis, 1993). For formal definitions and analytic solution methods for the SHS, one can refer to (Hespanha, 2004, Hespanha, 2005, Hespanha, 2006).

The state space of a SHS is a combination of a discrete state $q(t) \in Q$ and a continuous state $\mathbf{x}(t) \in \mathbb{R}^{n}$, where $Q$ is a finite set containing all the possible discrete modes of the system. Generally, a SHS is defined by the following items (Hespanha, 2006):

(1) A SDE that describes the evolution of the continuous state:

$$
d \mathbf{x}(t)=f(q(t), \mathbf{x}(t), t) d t+g(q(t), \mathbf{x}(t), t) d \mathbf{w}_{t},
$$

where $\mathbf{w}_{t}: \mathbb{R}^{+} \rightarrow \mathbb{R}^{k}$ is a k-dimensional Wiener process, $f: Q \times \mathbb{R}^{n} \times \mathbb{R}^{+} \rightarrow \mathbb{R}^{n}$, and $g: Q \times \mathbb{R}^{n} \times \mathbb{R}^{+} \rightarrow \mathbb{R}^{n \times k}$.

(2) Transition rates $\lambda_{j}(q(t), \mathbf{x}(t), t)$, and transition reset maps $\phi_{j}(q(t), \mathbf{x}(t), t), j \in 1, \ldots, l$, for all the $l$ transitions of the SHS among discrete modes. The probability of transition $j$ occurring in the time domain $[t, t+\Delta t)$ is: $\lambda_{j}(q(t), \mathbf{x}(t), t) \Delta t+o(\Delta t)$,

and if transition $j$ occurs at time $T$, the combined state of the SHS will be reset instantaneously by:

$(q(T), \mathbf{x}(T))=\phi_{j}\left(q\left(T^{-}\right), \mathbf{x}\left(T^{-}\right), T\right)$.

According to (Hespanha, 2006), given a test function $\psi(q(t), \mathbf{x}(t), t), \psi: Q \times \mathbb{R}^{n} \times \mathbb{R}^{+} \rightarrow \mathbb{R}$, which is twice continuously differentiable with respect to $\mathbf{x}$ and once continuously differentiable with respect to $t$, we have:

$\frac{d E[\psi(q(t), \mathbf{x}(t), t)]}{d t}=E[(L \psi)(q(t), \mathbf{x}(t), t)]$

where $(L \psi)(q, x, t)$ is known as the extended generator of SHS, and for $\forall(q, x, t) \in Q \times \mathbb{R}^{n} \times \mathbb{R}^{+}$

$$
\begin{aligned}
& (L \psi)(q, x, t):=\frac{\partial \psi(q, x, t)}{\partial x} f(q, x, t)+\frac{\partial \psi(q, x, t)}{\partial t} \\
& +\frac{1}{2} \operatorname{trace}\left(\frac{\partial^{2} \psi(q, x, t)}{\partial x^{2}} g(q, x, t) g(q, x, t)^{\prime}\right) \\
& +\sum_{j=1}^{l}\left(\psi\left(\phi_{j}(q, x, t), t\right)-\psi(q, x, t)\right) \lambda_{j}(q, x, t)
\end{aligned}
$$

where $\partial \psi / \partial t$ denotes the partial derivative of $\psi(q, x, t)$ with respect to $t, \partial \psi / \partial x$ and $\partial^{2} \psi / \partial x^{2}$ denote the gradient and Hessian matrix of $\psi(q, x, t)$ with respect to $x$, respectively.

Generally, test functions are defined as functions with respect to measures of the continuous state. Hereby, we define a family of test functions of the form:

$\psi_{q_{i}}^{(m)}(q, x)=\left\{\begin{array}{ll}x^{m} & q=q_{i} \\ 0 & q \neq q_{i}\end{array}\right.$,

where $m$ can be any natural number of interest. Let $\mu_{q_{i}}^{(m)}(t)$ denote the $m$-order conditional moment of the continuous state $x$, which is defined by

$$
\begin{aligned}
\mu_{q_{i}}^{(m)}(t) & :=E\left[\psi_{q_{i}}^{(m)}(q, x)\right] \\
& =E\left[x^{m}(t) \mid q(t)=q_{i}\right] \cdot \operatorname{Pr}\left\{q(t)=q_{i}\right\} .
\end{aligned}
$$

Then, by (4)(5) we can obtain a group of differential equations with respect to arbitrary order conditional moments $\mu_{q_{i}}^{(m)}(t), q_{i} \in Q, m \in \mathbb{R}^{+}$:

$d \mu_{q_{i}}^{(m)}(t)=E\left[(L \psi)_{q_{i}}^{(m)}(q, x)\right] \cdot d t$.

By solving the differential equations in (8), we can obtain knowledge about dynamics of the continuous state, based on which further analysis can be conducted. 


\section{SHS-BASED FAILURE BEHAVIOR MODELING}

\subsection{System description}

As shown in Figure 1, we consider a system suffering two dependent failure processes: soft failures caused jointly by continuous degradation and additional abrupt degradation increment due to random shocks, and hard failures caused by sudden overload from the same shock process. Dependence exists among the two process, that is, the arrival of a shock would bring an additional damage to the degradation process. Failures occur whenever one of the following two events happens:

i. the degradation process reaches its threshold, denoted by $H$;

ii. a shock whose magnitude exceeds a critical strength level, denoted by $D$, occurs.

Additional assumptions of this model include:

(1) The continuous degradation process is modeled by a Stochastic Differential Equation (SDE).

$$
d x(t)=\beta d t+b \cdot d w_{t},
$$

where $x(t)$ is the degradation measure, $\beta$ is a constant denoting the degradation rate, $w_{t}$ represents the Wiener process with $\sigma=1$, and $b$ is a constant.

(2) Random shocks arrive according to a Poisson process with intensity $\lambda$.

(3) The magnitude of each shock load, denoted by $W_{i}$, is an i.i.d. random variable following normal distribution $N\left(\mu_{W}, \sigma_{W}^{2}\right)$.

(4) To consider the dependence, we assume the arrival of each shock would bring a degradation increment of a constant $d$.

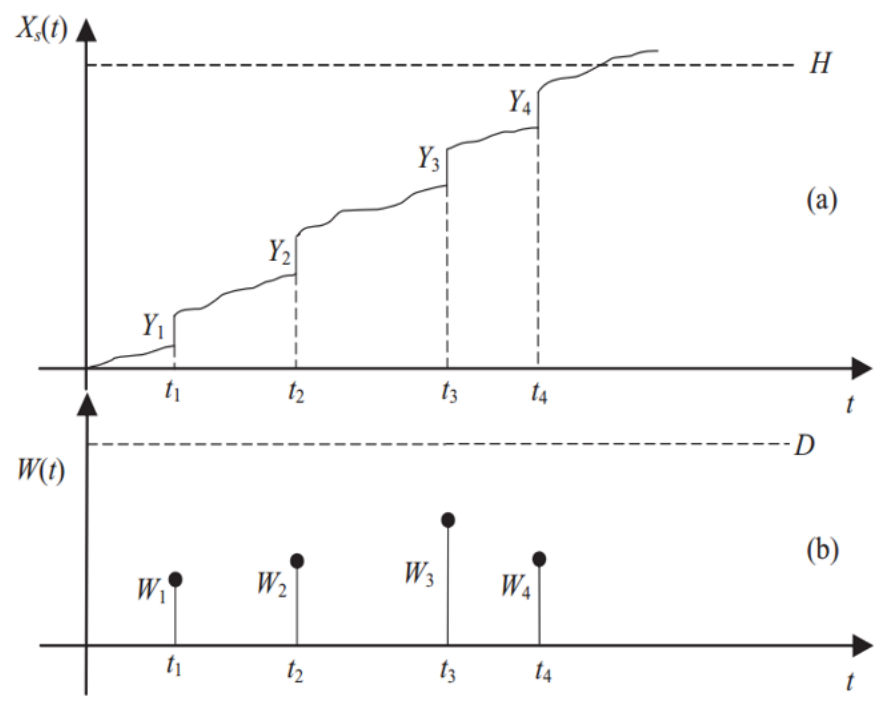

Figure 1. (a) Soft failure process (b) hard failure process

\subsection{SHS modeling of the system}

The SHS concerning the DCFP is described by the state-transition diagram in Figure 2. The system is a combination of (i) a continuous-time, discrete-state stochastic process $q(t) \in\left\{q_{1}, q_{2}\right\}$, where $q_{1}$ and $q_{2}$ are two possible states of the system. When $q=q_{1}$, the system is degrading according to the degradation process, while $q=q_{2}$ indicates that the system fails due to hard failures; and (ii) a continuous-time continuous-state stochastic process $x(t) \in \mathbb{R}$ that describes the degradation of the system over time. As shown in Figure 2, the system's discrete state starts from $q=q_{1}$, under which the dynamics of continuous state $x(t)$ is governed by (9). When a shock with magnitude less than $D$ arrives, system's discrete state remains at $q=q_{1}$, and the continuous state $x(t)$ will be reset: $x(t)=x(t)+d$, where $d$ is the damage caused by the random shock. When a shock with magnitude over $D$ arrives, the system transfers to the hard failure mode $q=q_{2}$ and the continuous state $x(t)$ is reset to zero.

The transition rates and transition reset maps of the above SHS are defined as follows:

$$
\begin{aligned}
& \lambda_{1}(q, x):= \begin{cases}\Phi\left(\frac{D-\mu_{W}}{\sigma_{W}}\right) \cdot \lambda & q=q_{1}, \\
0 & q=q_{2},\end{cases} \\
& \lambda_{2}(q, x):= \begin{cases}\left(1-\Phi\left(\frac{D-\mu_{W}}{\sigma_{W}}\right)\right) \cdot \lambda & q=q_{1}, \\
0 & q=q_{2} .\end{cases}
\end{aligned}
$$

$\phi_{1}(q, x):=\left(q_{1}, x+d\right)$,

$\phi_{2}(q, x):=\left(q_{2}, 0\right)$,

In this paper, we are interested in the evolution of degradation level $x$. So we define the test functions $\psi_{q_{i}}^{(m)}(q, x)$ in each discrete state $q_{i}, i \in\{1,2\}$ to be:

$\psi_{q_{1}}^{(m)}(q, x)=\left\{\begin{array}{ll}x^{m} & q=q_{1} \\ 0 & q \neq q_{1}\end{array}\right.$,
$\psi_{q_{2}}^{(m)}(q, x)=\left\{\begin{array}{ll}x^{m} & q=q_{2} \\ 0 & q \neq q_{2}\end{array}\right.$.

where $m$ can be any natural number of interest. We also define the conditional moments of the continuous state $x$ in each discrete state by:

$$
\begin{aligned}
& \mu_{q_{1}}^{(m)}(t):=E\left[x^{m}(t) \mid q(t)=q_{1}\right] \cdot \operatorname{Pr}\left\{q(t)=q_{1}\right\}, \\
& \mu_{q_{2}}^{(m)}(t):=E\left[x^{m}(t) \mid q(t)=q_{2}\right] \cdot \operatorname{Pr}\left\{q(t)=q_{2}\right\} .
\end{aligned}
$$

With the conditional moments defined above, we can obtain any order of conditional moments of interest by choosing $m$. It is obvious that $\mu_{q_{i}}^{(0)}(t)$ represents the probability that system's discrete state belongs to $q=q_{i}$. Substituting (9)(10)(11)(12) into (5), we have the extended generator of the SHS as: 


$$
\begin{aligned}
\left(L \psi_{q_{1}}\right)^{(m)}(q, x)= & \beta \frac{\partial \psi_{q_{1}}^{(m)}(q, x)}{\partial x}+\frac{1}{2} b^{2} \frac{\partial^{2} \psi_{q_{1}}^{(m)}(q, x)}{\partial x^{2}} \\
& +\lambda_{1}\left(\psi_{q_{1}}^{(1)}(q, x)+d \cdot \psi_{q_{1}}^{(0)}(q, x)\right)^{(m)} \\
& -\left(\lambda_{1}+\lambda_{2}\right) \psi_{q_{1}}^{(m)}(q, x), \\
\left(L \psi_{q_{2}}\right)^{(0)}(q, x)= & \lambda_{2} \cdot \psi_{q_{1}}^{(0)}(q, x) .
\end{aligned}
$$

According to (8) and (14), we have differential equations with respect to the conditional moments of $x$ as follows:

$$
\begin{aligned}
& \frac{d}{d t} \mu_{q_{1}}^{(m)}(t)=\beta m \mu_{q_{1}}^{(m-1)}(t)+\frac{1}{2} b^{2} m(m-1) \mu_{q_{1}}^{(m-2)}(t) \\
& \quad+\lambda_{1}\left(\sum_{k=0}^{m}\left(\begin{array}{c}
m \\
k
\end{array}\right) \mu_{q_{1}}^{(m-k)}(t) d^{k}\right)-\left(\lambda_{1}+\lambda_{2}\right) \mu_{q_{1}}^{(m)}(t), \\
& \frac{d}{d t} \mu_{q_{2}}^{(0)}(t)=\lambda_{2} \mu_{q_{1}}^{(0)}(t) .
\end{aligned}
$$

Because of the fact that when $q=q_{2}$, the continuous state $x(t)$ always equals to zero, in (15) we only concern the zero-order conditional moment at state $q_{2}$, i.e. the probability that the system fails due to a hard failure. It's clear that

$\mu_{q_{1}}^{(0)}+\mu_{q_{2}}^{(0)}=1$,

since the system must belong to one of the two discrete modes. By (15), we can obtain the following set of differential equations:

$$
\left[\begin{array}{c}
\dot{\mu}_{q_{1}}^{(0)} \\
\dot{\mu}_{q_{1}}^{(1)} \\
\dot{\mu}_{q_{1}}^{(2)}
\end{array}\right]=\left[\begin{array}{ccc}
-\lambda_{2} \mu_{q_{1}}^{(0)} & 0 & 0 \\
\beta+\lambda_{1} d & -\lambda_{2} & 0 \\
b^{2}+\lambda_{1} d^{2} & 2 \beta+2 \lambda_{1} d & -\lambda_{2}
\end{array}\right]\left[\begin{array}{c}
\mu_{q_{1}}^{(0)} \\
\mu_{q_{1}}^{(1)} \\
\mu_{q_{1}}^{(2)}
\end{array}\right] .
$$

By solving (16) and (17), we are able to find out (i) the probability of the SHS residing in each discrete mode, and (ii) the first-order and second-order moments of $x(t)$ conditioned on $q(t)$.

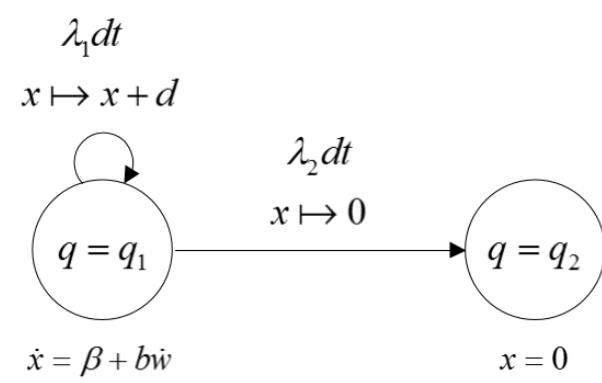

Figure 2. State-transition diagram for the SHS

\section{RELIABILITY CALCULATION}

Based on the conditional moments of $x(t)$ obtained in Sect. 3, the reliability of the DCFP can be approximated using FOSM method. Assume that at any time $t$, the conditional probability distribution of the degradation level $x(t)$ given $q=q_{1}$ is a normal distribution with mean $\mu_{x\left|q=q_{1}\right|}(t)$ and standard variance $\sigma_{x \mid q=q_{1}}(t)$. The $\mu_{x \mid q=q_{1}}(t)$ and $\sigma_{x \mid q=q_{1}}(t)$ can be calculated by

$$
\begin{aligned}
& \hat{\mu}_{x \mid q=q_{1}}(t)=E\left(x \mid q=q_{1}\right)=\frac{\mu_{q_{1}}^{(1)}(t)}{\mu_{q_{1}}^{(0)}(t)}, \\
& \begin{aligned}
\hat{\sigma}_{x \mid q=q_{1}}(t) & =\sqrt{D\left(x \mid q=q_{1}\right)} \\
= & \sqrt{E\left(x^{2} \mid q=q_{1}\right)-\left(E\left(x \mid q=q_{1}\right)\right)^{2}} \\
= & \sqrt{\frac{\mu_{q_{1}}^{(2)}(t)}{\mu_{q_{1}}^{(0)}(t)}-\left(\frac{\mu_{q_{1}}^{(1)}(t)}{\mu_{q_{1}}^{(0)}(t)}\right)^{2}} .
\end{aligned}
\end{aligned}
$$

Therefore, the reliability of the DCFP is

$$
\begin{aligned}
R(t) & =\operatorname{Pr}\left(q(t)=q_{1}, x(t)<H\right) \\
& =\operatorname{Pr}\left(q(t)=q_{1}\right) \cdot \operatorname{Pr}\left(x(t)<H \mid q(t)=q_{1}\right) \\
& =P\left(q(t)=q_{1}\right) \cdot \Phi\left(\frac{H-\hat{\mu}_{x \mid q=q_{1}}(t)}{\hat{\sigma}_{x \mid q=q_{1}}(t)}\right) \\
& =\mu_{q_{1}}^{(0)}(t) \cdot \Phi\left(\frac{H-\hat{\mu}_{x \mid q=q_{1}}(t)}{\hat{\sigma}_{x \mid q=q_{1}}(t)}\right) .
\end{aligned}
$$

The lower bound of reliability can also be estimated using Markov's inequality. According to Markov's inequality (Pishro-Nik, 2014), if $X$ is an nonnegative random variable and $a>0$, then

$\operatorname{Pr}(X \geq a) \leq \frac{E(X)}{a}$.

For the system we consider in this paper, the degradation level $x(t)$ is a nonnegative random variable when time $t$ is determined, and the threshold of soft failure $H>0$, then we have

$\operatorname{Pr}(x(t) \geq H) \leq \frac{E(x(t))}{H}$.

This inequality could be easily extended to the following inequality with respect to the conditional probability case:

$\operatorname{Pr}\left(x(t) \geq H \mid q=q_{1}\right) \leq \frac{E\left(x(t) \mid q=q_{1}\right)}{H}$.

Therefore, the lower bound of reliability is estimated by

$$
\begin{aligned}
R(t) & =\operatorname{Pr}\left(q(t)=q_{1}, x(t)<H\right) \\
& =\operatorname{Pr}\left(q(t)=q_{1}\right) \cdot \operatorname{Pr}\left(x(t)<H \mid q(t)=q_{1}\right) \\
& =\operatorname{Pr}\left(q(t)=q_{1}\right) \cdot\left[1-\operatorname{Pr}\left(x(t) \geq H \mid q(t)=q_{1}\right)\right] \\
& \geq \operatorname{Pr}\left(q(t)=q_{1}\right)\left[1-\frac{E\left(x(t) \mid q(t)=q_{1}\right)}{H}\right] \\
& =\mu_{q_{1}}^{(0)}(t)-\frac{\mu_{q_{1}}^{(1)}(t)}{H} .
\end{aligned}
$$




\section{NUMERICAL EXAMPLE}

A numerical example is carried out using the parameters listed in Table 1. To demonstrate the developed model, analytic solutions obtained by solving the differential equations in (17) are compared with results of Monte Carlo simulation, as shown in Figures 3-6. Results show that the dynamics of $\operatorname{Pr}\left(q=q_{1}\right)$, $E[x(t)]$ and $E\left[x^{2}(t)\right]$ are very accurately predicted by the SHS model. The estimated reliability by moment method is consistent with results by Monte Carlo simulation. The estimated lower bound provide a relatively conservative reliability estimation. Besides, the running time of Monte Carlo simulation is 2796.3 times more than that of the developed analytic approach (By Monte Carlo simulation: 548.08s, by analytic approach: $0.196 \mathrm{~s}$ ).

Table 1. Parameter values

\begin{tabular}{ll}
\hline Parameter & Value \\
\hline$H$ & $0.00125 \mu m^{3}$ \\
$\beta$ & $8.4823 \cdot 10^{-9} \mu m^{3}$ \\
$b$ & $6.0016 \cdot 10^{-10} \mu m^{3}$ \\
$d$ & $1.5 \mathrm{GPa}$ \\
$\lambda$ & $5 \cdot 10^{-3}$ \\
$\mu_{\mathrm{w}}$ & $1.2 \mathrm{GPa}$ \\
$\sigma_{\mathrm{w}}$ & $0.2 \mathrm{GPa}$ \\
$d$ & $1 \cdot 10^{-4} \mu \mathrm{m}^{3}$ \\
\hline
\end{tabular}

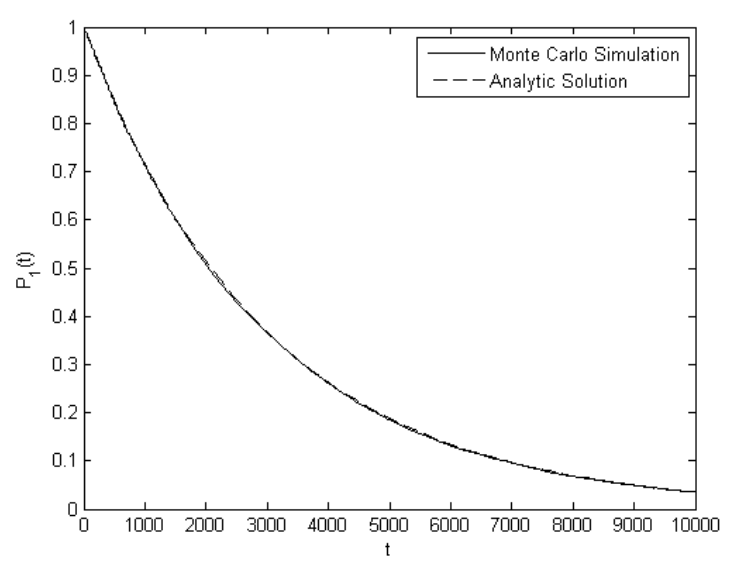

Figure 3. Results of $\operatorname{Pr}\left(q=q_{1}\right)$

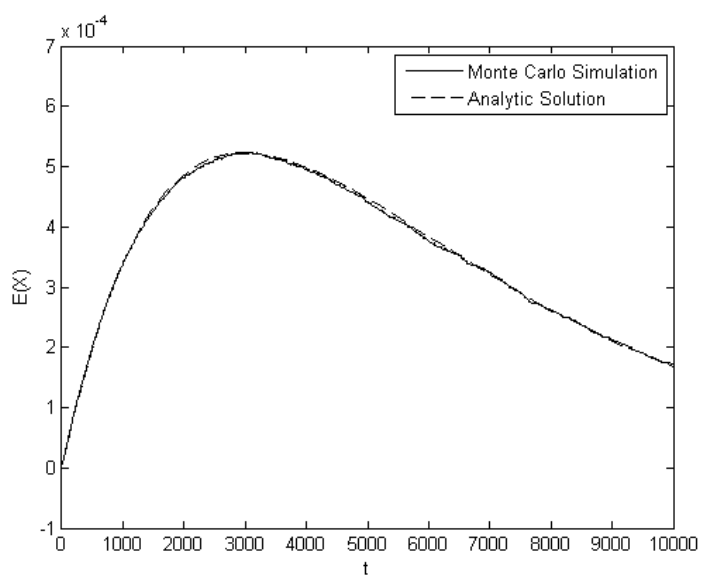

Figure 4. Results of $E[x(t)]$

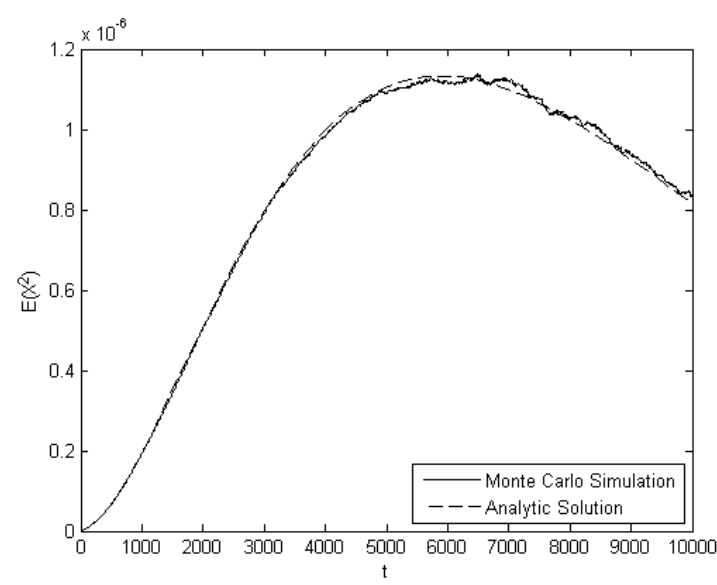

Figure 5. Results of $E\left[x^{2}(t)\right]$



Figure 6. $R(t)$ by MC simulation, the exact estimation of reliability: $R_{e}$, and the estimated lower boundary of reliability: $R_{l}$

\section{CONCLUSIONS}

In this paper a stochastic hybrid system characterizing systems subject to dependent competing failure processes are developed. The dependence is modeled by assuming the arrival of a random shock would lead to an abrupt increment of the degradation process. By solving the SHS-based DCFP model, dynamics of the conditional moments of degradation are obtained. Based on that, the lower boundary of system's reliability is estimated by Markov's inequality, and the exact reliability is approximated by moment method. Results show that the developed method provides accurate predictions of system's evolution with better computational performance.

\section{ACKNOWLEDGEMENT}

This work has been performed within the initiative of the Center for Resilience and Safety of Critical Infrastructures (CRESCI, http://cresci.buaa.edu.cn). This research is supported by the National Natural Science Foundation of China under grand number 61573043. 


\section{REFERENCES}

Bagdonavicius, V., Bikelis, A. \& Kazakevicuis, V. 2004. Statistical analysis of linear degradation and failure time data with multiple failure modes. Lifetime Data Analysis, 10, 6581.

Caballe, N.C., Castro, I.T., Perez, C.J. \& Lanza-Gutierrez, J.M. 2015. A condition-based maintenance of a dependent degradation-threshold-shock model in a system with multiple degradation processes. Reliability Engineering \& System Safety, 134, 98-109.

Davis, M.H.A. 1993. Markov models and optimization, London, UK, Chapman \& Hall.

Fan, J.J., Ghurye, S.G. \& Levine, R.A. 2000. Multicomponent lifetime distributions in the presence of ageing. Journal of Applied Probability, 37, 521-533.

Hespanha, J.P. 2004. Stochastic Hybrid Systems: Application to Communication Networks, Berlin, Heidelberg, Springer Berlin Heidelberg.

Hespanha, J.P. 2005. A model for stochastic hybrid systems with application to communication networks. Nonlinear Analysis: Theory, Methods \& Applications, 62, 1353-1383.

Hespanha, J.P. 2006. Modelling and analysis of stochastic hybrid systems. IEE Proceedings - Control Theory and Applications, 153, 520-535.

Huynh, K.T., Barros, A., Berenguer, C. \& Castro, I.T. 2011. A periodic inspection and replacement policy for systems subject to competing failure modes due to degradation and traumatic events. Reliability Engineering \& System Safety, 96, 497-508.

Huynh, K.T., Castro, I.T., Barros, A. \& Berenguer, C. 2012. Modeling age-based maintenance strategies with minimal repairs for systems subject to competing failure modes due to degradation and shocks. European Journal of Operational Research, 218, 140-151.

Jiang, L., Feng, Q. \& Coit, D.W. 2012. Reliability and maintenance modeling for dependent competing failure processes with shifting failure thresholds. IEEE Transactions on Reliability, 61, 932-948.

Jiang, L., Feng, Q. \& Coit, D.W. 2015. Modeling zoned shock effects on stochastic degradation in dependent failure processes. IIE Transactions, 47, 460-470.

Klutke, G.A. \& Yang, Y.J. 2002. The availability of inspected systems subject to shocks and graceful degradation. IEEE Transactions on Reliability, 51, 371-374.

Lemoine, A.J. \& Wenocur, M.L. 1985. On failure modeling. Naval Research Logistics Quarterly, 32, 497-508.

Li, W.J. \& Pham, H. 2005. Reliability modeling of multi-state degraded systems with multi-competing failures and random shocks. IEEE Transactions on Reliability, 54, 297-303.

Lin, Y.H., Li, Y.F. \& Zio, E. 2015. Integrating random shocks into multi-state physics models of degradation processes for component reliability assessment. IEEE Transactions on Reliability, 64, 154-166.

Peng, H., Feng, Q. \& Coit, D.W. 2010. Reliability and maintenance modeling for systems subject to multiple dependent competing failure processes. IIE Transactions, 43, 12-22.

Pishro-Nik, H. 2014. Introduction to probability, statistics, and random processes, Kappa Research, LLC.

Rafiee, K., Feng, Q. \& Coit, D.W. 2014. Reliability modeling for dependent competing failure processes with changing degradation rate. IIE Transactions, 46, 483-496. 